УДК 621.396 .946

\title{
ПАРАЛЛЕЛЬНОЕ ПОДАВЛЕНИЕ ПОМЕХ В МС-СDМА ПРИЕМНИКЕ С ПРОСТРАНСТВЕННО-ВРЕМЕННЫМ БЛОЧНЫМ КОДИРОВАНИЕМ
}

\author{
НУР МОХАМЕД ВАЛИ МОХАМАД ${ }^{1}$, СРИНАТА НАРАЯНАПА ${ }^{1}$, \\ ПАРТА ШАРАТИ МАЛИК ${ }^{1}$, НИТЯНАДАН ЛАКШМАНАН ${ }^{2}$ \\ ${ }^{1}$ VIT университет, \\ Индия, Веллуру, 632014, Тамилнад \\ ${ }^{2}$ Университет Пондичерри, \\ Индия, Калапет, Пондичерри
}

\begin{abstract}
Анотация. Множественный доступ с кодовым разделением и несколькими несущими MC-CDMA (multi carrier code division multiple access) является удобным методом для высокоскоростной передачи данных в канале с многолучевыми замираниями. Система MC-CDMA не может обрабатывать внезапные временные изменения канала, что приводит к тому, что поднесущие теряют ортогональность. Потеря ортогональности между поднесущими пользователя или нежелательная корреляция между кодами расширения спектра разных пользователей может привести к увеличению помех множественного доступа MAI (multiple access interference). Система MC-CDMA на основе пространственно-временных блочных кодов STBC (space time block code) выбрана для достижения полного разнесения и скорости передачи без знания информации о состоянии канала CSI (channel state information) для передатчика. Таким образом, в данной статье STBC вводится для передатчика с целью улучшения качества приема. Приемник на основе пространственно-временных блочных кодов с параллельным подавлением помех STBC-PIC (space time block code parallel interference cancellation) предложен для системы MC-CDMA. В предлагаемом STBC-PIC приемнике на каждом уровне подавления помех взвешенный сигнал другого пользователя вычитается из сигнала данного пользователя, тем самым уменьшая MAI и улучшая BER. Из результатов моделирования видно, что предлагаемый приемник превосходит приемники STBC с ортогональным полным дополняющим кодом (STBC-OCCC), STBC основанные на наименьшей среднеквадратической ошибке (STBC-MMSE) и STBC c обращением в нуль незначащих коэффициентов (STBC-ZF) с точки зрения уменьшения MAI.
\end{abstract}

Ключевые слова: множественный доступ с кодовым разделением и несколькими несущими; MC CDMA; многоканальный вход многоканальный выход; MIMO; помехи множественного доступа; пространственно-временное блочное кодирование; STBC; параллельное подавление помех

\section{1. ВВЕДЕНИЕ}

Множественный доступ с кодовым разделением и несколькими несущими МC-CDMA (multi carrier code division multiple access) есть одним из наиболее перспективных методов модуляции с несколькими несущими в технологии 4G [1]. МC-CDMA представляет собой комбинацию мультиплексирования с ортогональным частотным разделением каналов
OFDM (orthogonal frequency division multiplexing) и CDMA. Достоинства MC-CDMA включают максимальное использование спектра, легкую настройку под строгие условия канала без сложного детектирования и высокую стойкость к межсимвольной интерференции ISI (inter symbol interference) и замиранию, вызванному многолучевым распространением [2]. Это привлекательная техника для высокоскоростной передачи данных 


\section{БИБЛИОГРАФИЧЕСКИЙ СПИСОК}

1. Haitham, J. Taha; Salleh, M. F. M. Multi-carrier transmission techniques for wireless communication systems: A survey. WSEAS Trans. Commun., v.8, n.5, p.457-472, 2009. URI: http://www.wse- as.us/e-library/transactio-

ns/communications/2009/31-558.pdf.

2. Hara, S.; Prasad, R. Multicarrier Techniques for $4 G$ Mobile Communications. Boston London: Artech House, 2003.

3. Sarala, B.; Venkateswaralu, D. S.; Bhandari, B. N. Overview of MC CDMA PAPR reduction techniques. Int.

J. Distributed Parallel Systems, v.3, n.2, p.193-206, 2012. DOI: https://doi.org/10.5121/ijdps.2012.3217.

4. Fazel, K.; Kaiser, S. Multicarrier and Spread Spectrum Systems, 2nd ed. UK: Wiley Publishers, 2008. DOI: https://doi.org/10.1002/9780470714249.

5. Biglieri, Ezio; Calderbank, Robert; Constantinides, Anthony; Goldsmith, Andrea; Paulraj, Arogyaswami; Poor, H. Vincent. MIMO Wireless Communications. United Kingdom: Cambridge University Press, 2010.

6. D’Orazio, Leandro; Sacchi, Claudio; Fedrizzi, Riccardo; De Natale, Francesco G. B. An adaptive minimum-BER approach for multi-user detection in STBC-MIMO MC-CDMA systems, Proc. of IEEE GLOBECOM Conf., 26-30 Nov. 2007, Washington. DC, USA, IEEE, 2007, pp. 3427-3431. DOI: https://doi.org/10.1109/GLOCOM.2007.650.

7. Iraji, S.; Lilleberg, J. Interference cancellation for space-time block-coded MC-CDMA systems over multipath fading channels. Proc. of IEEE Vehicular Technology Conf., 6-9 Oct. 2003, Orlando, FL, USA. IEEE, 2003, v.2, p.1104-1108. DOI: https://doi.org/10.1109/VETECF.2003.1285192.

8. Portier, F.; Baudars, J.-Y.; Helard, J.-F. Performance of STBC MC-CDMA systems over outdoor realistic MIMO channels. Proc. of IEEE 60th Vehicular Technology Conf., 26-29 Sept. 2004, Los Angeles, CA, USA. IEEE, 2004, v.4, p.2409-2413. DOI: https://doi.org/10.1109/VETECF.2004.1400485.

9. Li, H.; Yangfe, W.; Tan, Z.; Cheng, S. Performance of space-time block-coded MC-CDMA system in multipath fading channel. Proc. of IEEE Int. Symp. on Microwave, Antenna, Propagation and EMC Technologies for Wireless Communications, 8-12 Aug. 2005, Beijing, China. IEEE, 2005, p.1546-1550. DOI: https://doi.org/10.1109/MAPE.2005.1618221.

$[10]=[6]$. D'Orazio, L., C. Sacchi, R. Fedrizzi, and F. G. De Natale (2007). An adaptive minimum-BER approach for multi-user detection in STBC-MIMO MC-CDMA systems, IEEE Global Telecommunications Conference, pp. 3427-3431. DOI: https://doi.org/10.1109/GLOCOM.2007.650.

11. Khan, M. A.; Umair, M.; Choudhry, M. A. S. Acceleration to LMS based STBC MC-CDMA receiver. Int. J. Scientific \& Engineering Research, v.4, n.8, p.925-929, 2013. !!! нет на сайте журнала https://www.ijser.org/ResearchPaperPublishing_August2013.aspx

12. Zhao, Fayong. PIC receiver for MIMO MC-CDMA systems based on LCRLSCMA. Proc. of 4th IEEE Conf. on Wireless Communications, Networking and Mobile Computing, WiCOM'08, 12-14 Oct. 2008, 
Dalian, China. IEEE, 2008, p.520-523. DOI: https://doi.org/10.1109/WiCom.2008.131.

13. Umair, M.; Khan, M. A.; Choudry, M. A. S. GA backing to STBC based MC-CDMA systems. Proc. of 4th IEEE Int. Conf. on Intelligent Systems Modelling and Simulation, 29-31 Jan. 2013, Bangkok, Thailand. IEEE, 2013, p.503-506. DOI: https://doi.org/10.1109/ISMS.2013.26.

14. Ajra, H.; Hasan, Mohamad Z.; Islam, Md. S. BER analysis of various channel equalization schemes of a QO-STBC encoded OFDM based MIMO CDMA system. Int. J. Computer Network and Information Security, v.6, n.3, p.30-36, 2014. DOI: https://doi.org/10.5815/ijenis.2014.03.04.

15. Senthilkumar, G.; Amutha, R. Capacity enhancement of MCCDMA systems through MAI cancellation using switched interleaving technique and correlation reconstruction based MRC with diversity gain. Int. J. Soft Comput. Eng., v.4, n.1, p.83-87.

16. El Sharef, Semi; Khedr, Mohamed; Badran, Ehab F. Enhancing MC-CDMA system using rotated quasi-orthogonal STBC in wireless channels. Proc. of IEEE Wireless Advanced Conf., 25-27 June 2012, London, UK. IEEE, 2012, p.110-114. DOI: https://doi.org/10.1109/WiAd.2012.6296544.

17. Glisic, Savo G. Advanced Wireless Communications: 4G Technologies. Chichester, England: John Wiley and Sons Ltd., 2005.

18. Cai, Yunlong; de Lamare, Rodrigo C.; Le Ruyet. Didier. Transmit processing techniques based on switched interleaving and limited feedback for interference mitigation in multiantenna MC-CDMA systems. IEEE Trans. Vehicular Technol., v.60, n.4, p.1559-1570, 2011. DOI: https://doi.org/10.1109/TVT.2011.2109744. 